\title{
The differential effects of statins on the metastatic behaviour of prostate cancer
}

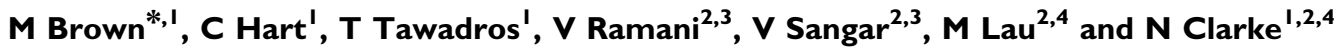 \\ 'Genito Urinary Cancer Research Group, University of Manchester, Paterson Institute for Cancer Research, Manchester Academic Health Science Centre, \\ The Christie NHS Foundation Trust, Wilmslow Road, Withington, Manchester M20 4BX, UK; ${ }^{2}$ Department of Urology, The Christie NHS Foundation \\ Trust, Wilmslow Road, Manchester M20 4BX, UK; ${ }^{3}$ Department of Urology, University Hospital of South Manchester NHS Trust, Manchester M23 $9 L T$, \\ UK; ${ }^{4}$ Department of Urology, Salford Royal NHS Foundation Trust, Stott Lane, Manchester M6 8HD, UK
}

\begin{abstract}
BACKGROUND: Although statins do not affect the incidence of prostate cancer (CaP), usage reduces the risk of clinical progression and mortality. Although statins are known to downregulate the mevalonate pathway, the mechanism by which statins reduce CaP progression is unknown.

METHODS: Bone marrow stroma (BMS) was isolated with ethical approval from consenting patients undergoing surgery for nonmalignant disease. PC-3 binding, invasion and colony formation within BMS was assessed by standardised in vitro co-culture assays in the presence of different statins.

RESULTS: Statins act directly on PC-3 cells with atorvastatin, mevastatin, simvastatin ( $1 \mu \mathrm{M})$ and rosuvastatin ( $5 \mu \mathrm{M})$, but not pravastatin, significantly reducing invasion towards BMS by an average of $66.68 \%$ (range $53.93-77.04 \% ; P<0.05$ ) and significantly reducing both number $(76.2 \pm 8.29$ vs $122.9 \pm 2.48 ; P=0.0055)$ and size $\left(0.2 \pm 0.0058 \mathrm{~mm}^{2}\right.$ vs $\left.0.27 \pm 0.012 \mathrm{~mm}^{2} ; P=0.0019\right)$ of colonies formed within BMS. Statin-treated colonies displayed a more compact morphology containing cells of a more epithelial phenotype, indicative of a reduction in the migrational ability of PC-3 cells. Normal PC-3 phenotype and invasive ability was recovered by the addition of geranylgeranyl pyrophosphate (GGPP).

CONCLUSION: Lipophilic statins reduce the migration and colony formation of PC-3 cells in human BMS by inhibiting GGPP production, reducing the formation and the spread of metastatic prostate colonies.

British Journal of Cancer (2012) 106, 1689-1696. doi:I0.1038/bjc.2012.138 www.bjcancer.com

Published online 24 April 2012

(c) 2012 Cancer Research UK
\end{abstract}

Keywords: prostate cancer; metastasis; statins; bone marrow stroma

Prostate cancer $(\mathrm{CaP})$ is the second most common malignancy in men worldwide, with 910000 new cases diagnosed in 2008 (Ferlay et al, 2010). CaP has a predilection to metastasise to the bone marrow stroma (BMS), and development of CaP bone metastases almost invariably result in CaP-related mortality (George, 1988). The metastatic process is a complex, multistep process, which can be modelled with modified in vitro invasion chambers utilising human primary BMS (Lang et al, 1998; Scott et al, 2001; Hart et al, 2005). These in vitro models not only allow the determination of the mechanism of $\mathrm{CaP}$ metastasis to the BMS but enable the elucidation of how therapeutic agents may interfere with the metastatic process.

Recent evidence links lipid metabolism and statin use with the behaviour of CaP. Large scale epidemiological data showing lower rates of $\mathrm{CaP}$ progression in patients taking statins (Platz et al, 2006) have been consolidated by reports showing that $\mathrm{CaP}$ incidence in screened (Murtola et al, 2010) and non-screened (Breau et al, 2010) populations is reduced in men taking these drugs and that individuals with lower cholesterol levels had lower rates of high risk disease (Platz et al, 2008). Further clinical data from histological analysis of large case after prostatectomy series

*Correspondence: Dr MD Brown; E-mail: mbrown@picr.man.ac.uk Received 23 January 2012; revised 13 March 2012; accepted 21 March 2012; published online 24 April 2012 showed less aggressive features in men taking statins (Loeb et al, 2010) and in patients undergoing radiotherapy for CaP indices of $\mathrm{CaP}$ treatment failure were reduced in men taking statins, especially in those with high risk features (Gutt et al, 2010).

The basis for these observations is poorly understood but the effects probably relate to the pleiotropic actions of 3-hydroxy-3methylglutaryl-CoA (HMG-CoA) inhibitors on cellular behaviour, cell-cell interaction and cellular motility in relation to lipid metabolism (Stancu and Sima, 2001). To better understand this process, we have studied the effects of different statins in our inhouse, well-categorised models of $\mathrm{CaP}$ behaviour, evaluating the differential effects of specific statins on cellular binding, migration and early cellular survival in human BMS.

\section{MATERIALS AND METHODS}

\section{Cell culture}

PC-3 (ATCC-LGC, Teddington, UK) cells were cultured in HAM'SF12 media supplemented with $7 \%$ foetal calf serum (FCS) and $2 \mathrm{~mm}$ L-glutamine at $37{ }^{\circ} \mathrm{C}, 5 \% \mathrm{CO}_{2}$ in air. PC3-GFP were cultured as for PC-3 except for addition of $0.15 \mathrm{mg} \mathrm{ml}^{-1}$ hygromycin B. Human BMS was obtained from volunteers undergoing surgery for benign disease and cultured according to Coutinho et al (1993). Briefly, $2 \times 10^{6}$ cells ml $^{-1}$ in long-term bone marrow culture medium 
(LTBCM) (Iscove's modified Dulbecco's medium at $350 \mathrm{mOsm}$, $10 \%$ foetal calf serum, $10 \%$ horse serum, $5 \times 10^{-7} \mathrm{M}$ hydrocortisone) were grown at $33{ }^{\circ} \mathrm{C}$ in $5 \% \mathrm{CO}_{2}$ in air for $4-5$ weeks until haematopoietically active areas were observed. Bone marrow endothelial cells (BMEC) were cultured in LTBCM conditioned by BMS on fibronectin- $\left(50 \mathrm{mg} \mathrm{ml}^{-1}\right.$ in PBS) treated flasks. All cell lines were verified by the Paterson Institute for Cancer Research tissue typing service.

\section{Statins and metabolites}

Atorvastatin, rosuvastatin (Discovery Fine Chemicals Ltd, Dorset, UK), mevastatin, and simvastatin (Sigma-Aldrich, Poole, UK) were dissolved in dimethyl sulphoxide (DMSO) and pravastatin (SigmaAldrich) was dissolved in water at concentrations of $100 \mathrm{~mm}$. Activated simvastatin was also assessed. Briefly, $4 \mathrm{mg}$ of simvastatin was dissolved in $100 \mu \mathrm{l}$ of ethanol to which $150 \mu \mathrm{l}$ of $0.1 \mathrm{~N}$ $\mathrm{NaOH}$ was added and incubated at $50{ }^{\circ} \mathrm{C}$ for $2 \mathrm{~h}$. The $\mathrm{pH}$ was brought to 7.0 by $\mathrm{HCl}$ and diluted to a final concentration of $100 \mathrm{~mm}$ before storing at $4{ }^{\circ} \mathrm{C}$. All statins were used at final nontoxic concentrations as defined by trypan blue exclusion. All metabolites were purchased from Sigma-Aldrich. Geranylgeranyl pyrophosphate (GGPP) and farnesyl pyrophosphate (FPP) were dissolved in methanol at a final concentration of $1 \mathrm{mg} \mathrm{m}^{-1}$. Cholesterol was dissolved in chloroform at $100 \mathrm{~mm}$. Mevalonate was made up in water at a stock concentration of $10 \mathrm{mg} \mathrm{ml}^{-1}$ $(40 \mathrm{~mm})$

\section{Binding assay}

Prostate epithelial cell (PEC) binding to BMS was determined as previously described (Scott et al, 2001); $5 \times 10^{4}$ PC3-GFP cells, treated with statin or DMSO vehicle control, were added to confluent BMS in a 96-well plate. Wells were washed and fluorescence was read using a FLUOstar OPTIMA spectrometer (BMG Labtech, Aylesbury, UK). Binding was expressed as percent of pre-washed total fluorescence.

\section{Invasion assay}

Prostate epithelial cell invasion towards BMS was measured as previously described (Hart et al, 2005); invasion towards statinpretreated BMS or in the presence of statins was assessed. The FluoroBlok (BD Biosciences, Oxford, UK) tumour cell invasion system used phenol red-free RPMI 1640 media and FluoroBlok cell culture inserts coated with $100 \mu \mathrm{l}$ of Matrigel (BD Biosciences) (1:25 dilution with RPMI 1640) above chemoattractant (BMS or tissue culture plastic (TCP)). $2 \times 10^{5}$ PC3-GFP in RPMI $1640 / 0.1 \%$ BSA were added to each FluoroBlok insert and the co-cultures incubated at $37^{\circ} \mathrm{C}, 5 \% \mathrm{CO}_{2}$ for $18 \mathrm{~h}$. Bone marrow stroma was pretreated daily with the different statins. PC-3 cells were treated with statins $30 \mathrm{~min}$ before addition. Final readings utilised the FLUOstar OPTIMA spectrometer. Recovery assays were conducted as above or in the presence of a confluent BMEC cell layer barrier (Hart et al, 2005). PC3-GFP cells were treated with $10 \mu \mathrm{M}$ GGPP, $100 \mu \mathrm{m}$ mevalonate, $16 \mu \mathrm{m}$ cholesterol or $10 \mu \mathrm{m}$ FPP immediately before the additions of statins and adding to the invasion chamber.

\section{Co-culture colony assay}

Prostate epithelial cell growth in BMS co-culture was measured as previously described (Lang et al, 1998); confluent primary human BMS, pretreated with statins for $60 \mathrm{~min}$, was seeded with $500 \mathrm{PC}-3$ cells. Statins were refreshed daily for 8 days before $4 \%$ paraformaldehyde fixation. Co-cultures were permeabilised using ice-cold methanol and blocked with $10 \%$ rabbit serum, followed by $0.3 \%$ hydrogen peroxide. Co-cultures were incubated with mouse anti-human pan-cytokeratin at 1:200 (Sigma-Aldrich) followed by biotinylated rabbit anti-mouse 1:400 (DAKO Ltd., Cambridge, UK). A complex of avidin DH and biotinylated horseradish peroxidase $\mathrm{H}$ (Vector Laboratories, Peterborough, UK) was then added and developed with DAB substrate.

\section{Clonogenic assay}

Five hundred PC-3 cells were treated at day 1 and day 7 with statins, before methanol acetone fixation and staining with crystal violet on day 14; colonies ( $>32$ cells) were then counted using a standard microscope graticule.

\section{Statistics}

Values are presented as mean \pm s.e. Assays were compared using the two-tailed Student's $t$-test with significance set at $P<0.05$.

\section{RESULTS}

\section{Reduction of BMS invasion by lipophilic statins}

We have previously shown that BMS is the most potent chemoattractant for metastatic PECs and that the metastatic process can be modelled using primary human BMS co-cultures (Hart et al, 2005; Brown et al, 2006). We therefore addressed three different questions: do statins affect the BMS microenvironment (Figure 1A), the PECs directly (Figure 1B) or the interaction between BMS and PECs (Figure 1C). Statins did not affect the ability of BMS to induce PEC invasion; although atorvastatin and simvastatin treatment of BMS reduced invasion as compared with the controls ( $84 \pm 8.8 \% ; P=0.18806$ and $80 \pm 10.57 \% ; P=0.14755)$, this did not reach significant levels (Figure 1A). All statins, except pravastatin, induced a similar and marked reduction in PEC invasion towards BMS $(P>0.05)$, averaging $66.68 \%$ of control (range $54-77 \% ; P<0.05$ ). Bone marrow stroma pretreatment (Figure 1C) did not provide additional benefit to treating PECs alone $(58.86 \% ; P>0.05$ of $P C-3$ 's invading compared with controls), suggesting a direct effect on the PC-3 cells and not on the BMS 'soil'.

The effect of statins was more significant in the presence of a confluent BMEC/Matrigel barrier, which models the blood BMS barrier. Both $5 \mu \mathrm{m}$ rosuvastatin and $1 \mu \mathrm{M}$ simvastatin (Figure 1D) completely inhibited direct invasion towards BMS (9.37\% vs $100 \%, P=0.0201$ and $25.6 \%$ vs $100 \%, P=0.0496$ for rosuvastatin and simvastatin vs BMS + vehicle control, respectively), with $5 \mu \mathrm{M}$ rosuvastatin reducing invasion to levels similar to TCP $(P=0.21212)$. Activation of simvastatin according to manufacturer's instructions had no effect on the ability of simvastatin to inhibit transendothelial invasion towards BMS $(13.5 \% \pm 7.1 \%$ and $14.8 \% \pm 12.8 \%$, respectively; $P=0.142)$ (Supplementary Figure 1).

\section{Effect of statins on PEC binding to BMS}

When malignant PECs enter the BMS, they migrate towards and bind to niches within the BMS before proliferating and forming viable colonies (Brown et al, 2010). We sought to assess the inhibitory effect of statins on this process. Incubation of PECs with statins for up to $120 \mathrm{~min}$ did not alter the ability of PECs to bind to BMS (Figure 2).

\section{Reduction of PEC colony formation in BMS by lipophilic statins}

Previous studies have documented the antiproliferative effect of high-dose statin treatment of PECs. However, the antiproliferative effect of statins on PEC within the lipid-rich environment of the BMS is unknown. We therefore compared the effect of statins on 
A
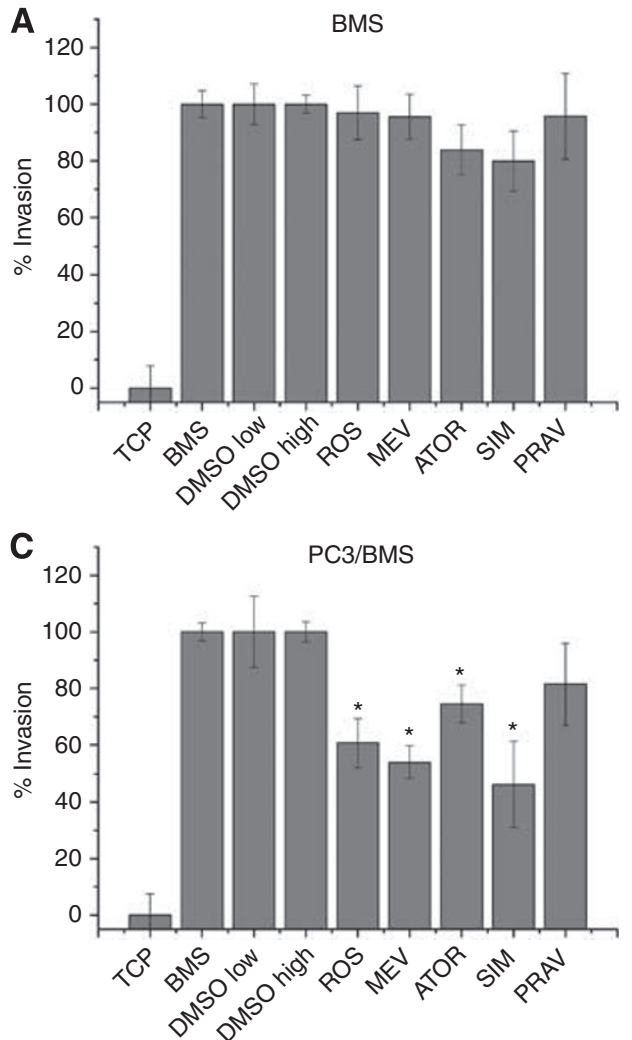

B

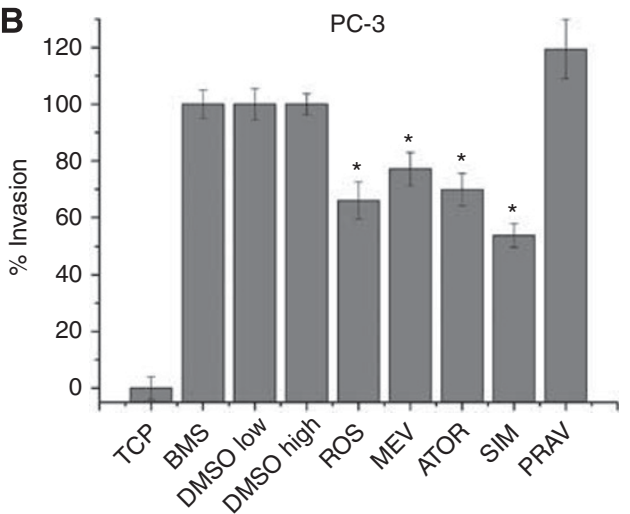

D

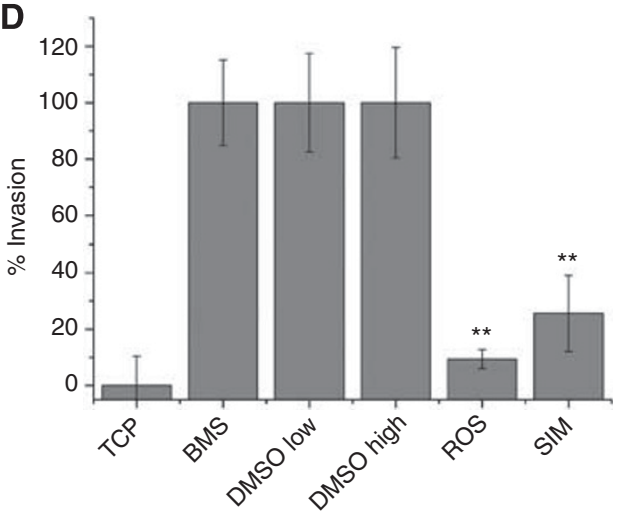

Figure I Statins directly affect the ability of CaP to invade towards BMS. (A) Bone marrow stroma treated with statins (rosuvastatin (ROS), mevastatin (MEV), atorvastatin (ATOR), simvastatin (SIM) or pravastatin (PRAV)) for 7 days before washing and using as an invasion stimulus in a Boyden chamber assay in the absence of statins. (B) PC-3 treated with statins 30 min before adding to the top of the Boyden chamber invasion assay. (C) PC-3 cells were pretreated for 30 min before invasion assay in the presence of statins towards BMS previously treated with statins for 7 days. Invasion normalised to TCP and to either BMS (PRAV) or DMSO vehicle controls (atorvastatin, MEV, ROS, SIM); $n=3$. (D) PC-3 treated with either I $\mu \mathrm{M}$ SIM or $5 \mu \mathrm{M}$ ROS 30 min before adding to the top of the Boyden chamber invasion assay, which contained a confluent BMEC layer on top of a Matrigel layer. * denotes $P<0.05$ from vehicle control. *** denotes $P>0.05$ between statins.
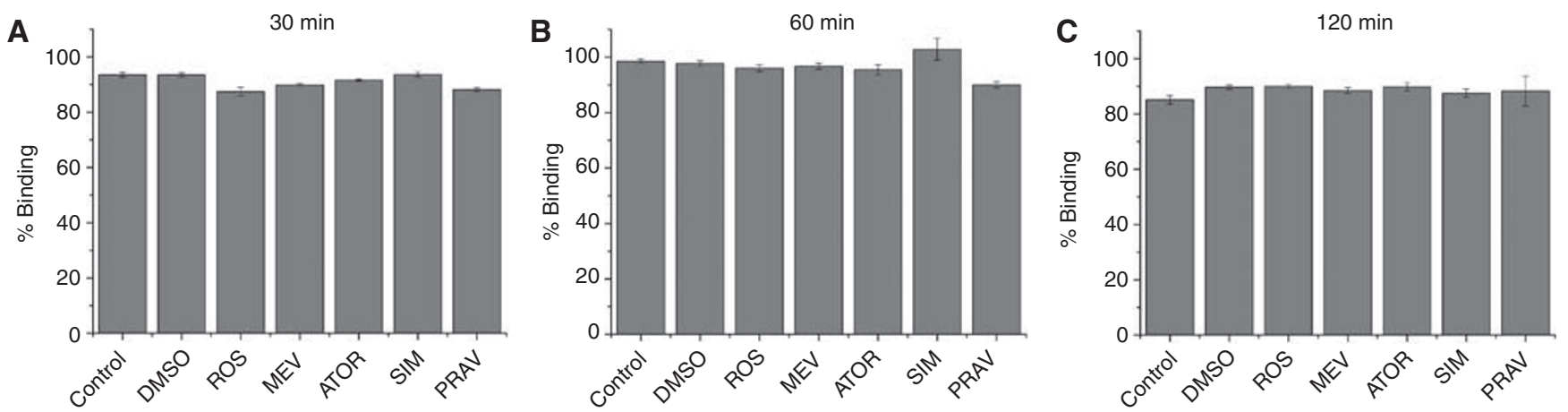

Figure 2 Statins do not inhibit PEC binding to human BMS. PC3-GFP cells were incubated with I $\mu \mathrm{M}$ atorvastatin, SIM, MEV, $5 \mu \mathrm{M}$ ROS or I0 $\mu \mathrm{M}$ PRAV for (A) 30, (B) 60 or (C) 120 min before binding to confluent cultures of BMS. After 60 min, unbound cells were washed off and the mean well fluorescence was determined using a FLUOstar OPTIMA spectrometer. Data expressed as a percentage of the maximum well fluorescence pre-washing; $n=3$.

PEC colony formation and proliferation in isolation with clonogenic assay or in BMS co-culture.

Pravastatin, at doses up to $100 \mu \mathrm{M}$, had no effect on PC-3 colony formation or cellular proliferation in either clonogenic or BMS coculture assays (Figure 3A). However, the lipophilic statins and rosuvastatin all had a significant effect on colony formation in both assays and cellular proliferation. Lipophilic statins and rosuvastatin significantly reduced the number of colonies to a similar degree in clonogenic assays and BMS co-culture (Table 1), with simvastatin being the most potent (reduction of $75.44 \%$ and
$49.44 \%$, respectively). Bone marrow stroma colonies were smaller than controls $\left(0.19 \pm 0.025 \mathrm{~mm}^{2}, 0.21 \pm 0.03 \mathrm{~mm}^{2}, 0.21 \pm 0.03 \mathrm{~mm}^{2}\right.$, $\left.0.19 \pm 0.03 \mathrm{~mm}^{2} ; P<0.05\right)$ resulting in a reduction of the total PEC area $\left(12.8 \pm 3.34 \mathrm{~mm}^{2}, \quad 20.7 \pm 4.5 \mathrm{~mm}^{2}, \quad 18.68 \pm 4.1 \mathrm{~mm}^{2}\right.$, $14 \pm 3.8 \mathrm{~mm}^{2}$ ) in co-cultures treated with rosuvastatin, atorvastatin, mevastatin and simvastatin, respectively (Figure 3B). There was no difference $(P>0.05)$ in the colony size, number or overall PEC load between the four statins.

Typically, PC-3 colonies in BMS co-culture formed large diffuse colonies of migrating PECs within the BMS. Cells displayed a 
mesenchymal morphology, being teardrop shaped, with ruffled leading edges of lamellipodia in the absence of therapeutic agents or in the presence of DMSO vehicle control (Figure 3C, control). The lipophilic statins induced significant morphological changes in the BMS colonies. Prostate epithelial cell colonies were more tightly packed, containing fewer cells than controls. At a higher magnification they displayed differing morphology compared with the mesenchymal morphology seen in control co-cultures, with
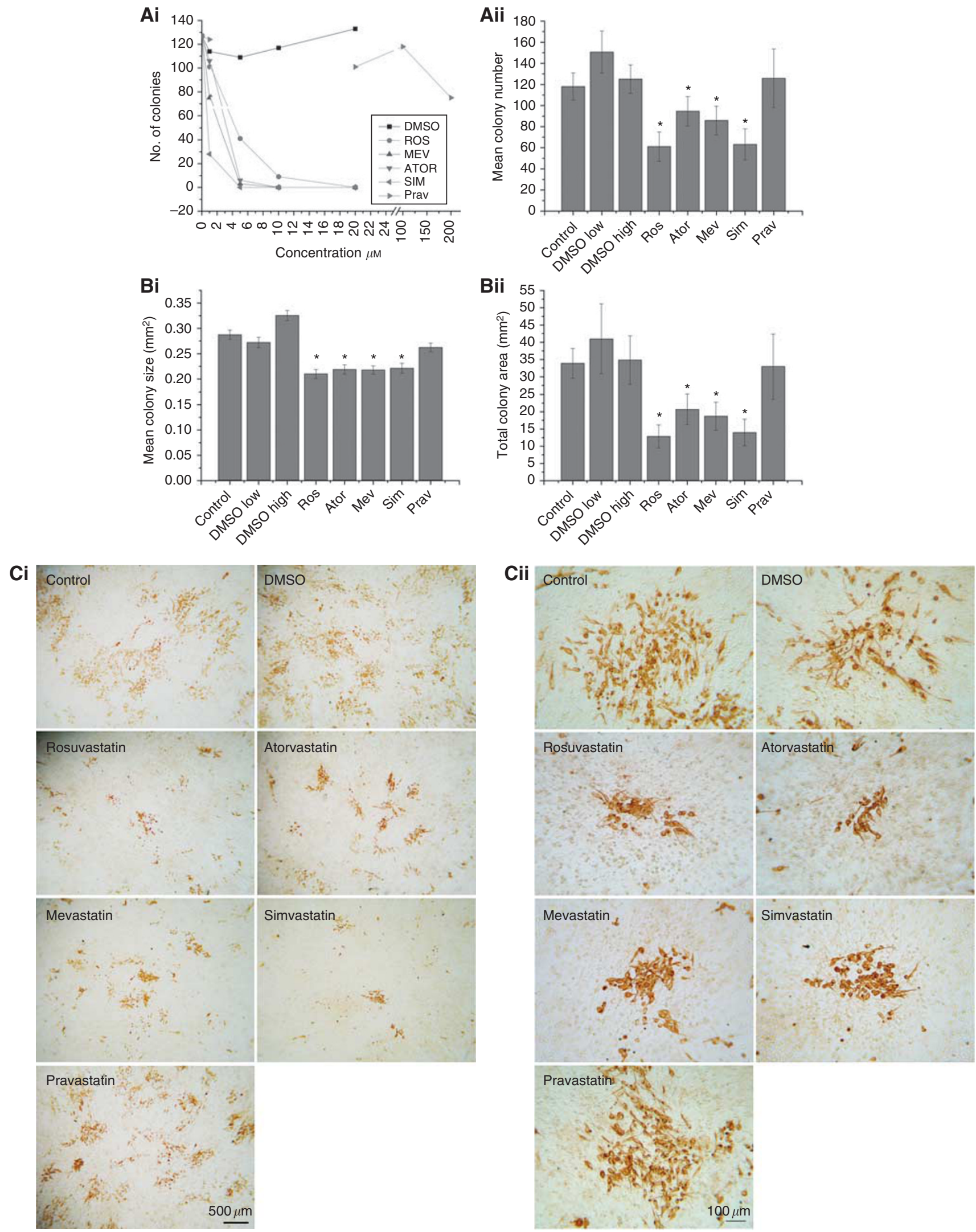
Table I Effect of stains on prostate epithelial colony formation in BMS co-culture

\begin{tabular}{lcccc}
\hline & $\begin{array}{c}\text { Mean colony } \\
\text { number }\end{array}$ & s.e. & $\begin{array}{c}\text { Fold } \\
\text { reduction }\end{array}$ & P-value \\
\hline No drug control & 118 & 12.84 & & \\
DMSO low control & 150.7 & 19.97 & & \\
DMSO high control & 125 & 13.54 & & \\
Rosuvastatin & 61.2 & 13.76 & 2.04 & 0.0095 \\
Atorvastatin & 94.6 & 13.91 & 1.59 & 0.055 \\
Mevastatin & 85.8 & 13.56 & 1.76 & 0.0315 \\
Simvastatin & 63.2 & 4.72 & 2.38 & 0.0117 \\
Pravastatin & 125.8 & 27.79 & 0.94 & 0.7928 \\
\hline
\end{tabular}

Abbreviations: $\mathrm{BMS}=$ Bone marrow stroma; $\mathrm{DMSO}=$ dimethyl sulphoxide; $\mathrm{PC}-3$ colony formation after statin treatment in BMS co-culture assay. PC-3 cells were treated with I $\mu \mathrm{M}$ atorvastatin, mevastatin, simvastatin, $5 \mu \mathrm{M}$ rosuvastatin or $10 \mu \mathrm{M}$ pravastatin daily for 8 days in BMS co-culture. Colonies $>32$ cells were counted. Table I shows the mean number of colonies, the fold reduction in numbers as compared with the relevant vehicle controls and the significant difference between treatments and their respective vehicle controls.
PECs being more rounded, without obvious lamellipodia at the cell's leading edge. Although some cells retained their teardrop appearance, the leading edge did not possess lamellipodia and the tails were distorted, appearing longer, wider and curved (Figure 3C). These morphological effects were not seen with pravastatin.

\section{Mechanism of statin inhibition of invasion}

Statins act by blocking the conversion of HMG-CoA to mevalonic acid through inhibition of HMG-CoA reductase (Figure 4A). We therefore sort to recover the invasion by supplying the downstream metabolites mevalonate, cholesterol or the prenylating agents GGPP and FPP. Treating PC-3-GFP cells with either GGPP or mevalonate had no significant effect on their ability to invade towards BMS $(P=0.259$ and 0.619 , respectively). Both cholesterol and FPP reduced PC3-GFP invasion towards BMS, but this reduction was not significant $(75.51 \% \pm 2.25 \% ; P=0.158$ and $54.9 \% \pm 8.24 \% ; P=0.056$, respectively). PC3-GFP cells were treated with mevalonate, GGPP, FPP or cholesterol before treatment with
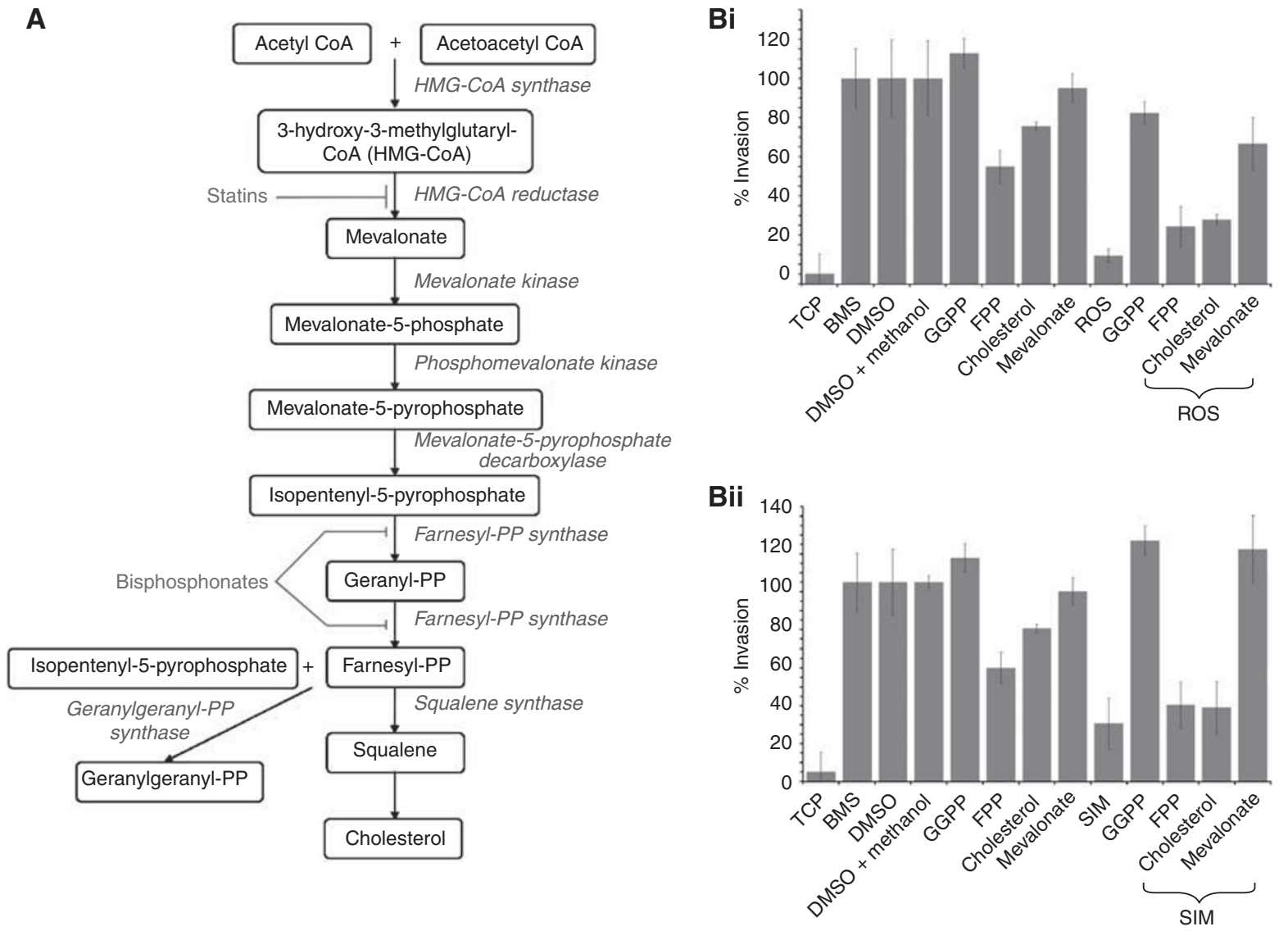

Figure 4 Geranylgeranyl pyrophosphate recovers statin-inhibited invasion towards BMS. (A) Diagrammatic representation of the mevalonate pathway highlighting the metabolic blocks of statins and bisphosphonates. (B) Histogram depicting the effect of the addition of the mevalonate pathway metabolites (mevalonate, FPP, GGPP or cholesterol) downstream of the statin block in a BMS co-culture assay utilising a BMEC barrier layer. (Bi) Invasion recovery assay in the presence of $5 \mu \mathrm{M}$ ROS. (Bii) Invasion recovery assay in the presence of I $\mu \mathrm{M}$ SIM.

Figure 3 Statins inhibit both colony formation in mono and BMS co-culture and spread in BMS co-culture. (Ai) Statin toxicity on PC-3 cells was determined by |4-day clonogenic assays with statin refreshed at day 7 . Colonies of $>32$ cells were counted. (Aii) Confluent BMS was pretreated for 60 min with statins before the addition of 500 PC-3 cells. Statins were refreshed daily for 8 days before co-cultures were fixed and PECs stained with pan-cytokeratin DAB. Histogram shows the number of prostate colonies formed, (Bi) histogram showing the mean size of colonies formed in BMS co-culture and (Bii) histogram showing the total prostate colony area formed in BMS co-culture in the presence of statins; * denotes $P<0.05$ from vehicle control. (Ci) Photomicrographs of typical prostate colonies formed in BMS co-culture in the presence of statins. (Cii) High magnification photomicrographs of a single colony formed in bone marrow stromal co-culture in the presence of media alone, DMSO, ROS, ATOR, MEV, SIM, PRAV; $n=3$. 
either $1 \mu \mathrm{m}$ simvastatin or $5 \mu \mathrm{m}$ rosuvastatin, and invasion through a BMEC/ Matrigel barrier towards untreated BMS was assessed. The addition of mevalonate to the invasion assay completely restored/enhanced the invasion towards BMS $(66.67 \% \pm 13.26 \%$; $P=0.1126$ and $117.42 \% \pm 17.79 \% ; P=0.0033$ for rosuvastatin and simvastatin, respectively). However, of the addition of downstream metabolites, only GGPP was able to completely restore rosuvastatin-treated invasion towards BMS $(82.42 \pm 5.52 \% ; P=0.229)$. Addition of GGPP to simvastatin-treated cells resulted in an enhanced invasion as compared with the statin-free controls $(122 \pm 7.83 \% ; P=0.0185)$. Addition of either FPP or cholesterol did not affect the ability of rosuvastatin or simvastatin to inhibit invasion towards BMS. Activation of simvastatin had no effect on the recovery profile, with both GGPP $(111.5 \pm 8.59 \% ; P=0.9079)$ and mevalonate $(67.04 \pm 8.03 \% ; P=0.0547)$ restoring the invasive ability of PC3-GFP cells towards BMS $(P=0.1391$ and $P=0.2267$ comparing activated to not activated simvastatin in the presence of GGPP or mevalonate, respectively) (Supplementary Figure 2).

\section{DISCUSSION}

This is the first study to report the comparative effects of different statins on CaP cellular migration towards and within human BMS. The results demonstrate a clear effect on $\mathrm{CaP}$ migration towards and through BMS and on malignant PEC's ability to grow clonally in that location. However, this effect was limited to the lipophilic statins and was not seen with the hydrophilic statin, pravastatin. The differential effect of the two basic subtypes of this class of drug has been well described; lipophilic statins diffuse across cellular membranes and exert their metabolic effects in the liver and other tissues; hydrophilic statins require active transport across the cell membrane in order to exert their actions intracellularly. This action of hydrophilic agents is therefore predominantly hepatic and not peripheral (Stancu and Sima, 2001; Garwood, 2010) and this fact has significant consequences for the effects observed in neoplasms such as breast and other cancers (Campbell et al, 2006; Kotamraju et al, 2007; Koyuturk et al, 2007). This differential action was clearly evident in the results presented herein and may be an important consideration when interpreting data from population studies of the effect of statins or when planning statin-based prevention trials in CaP.

Epidemiological studies have shown that although statin use does not affect $\mathrm{CaP}$ incidence, it does reduce the risk of clinical progression and CaP-related mortality; the mechanisms of this are unknown. Statins can induce apoptosis in a range of malignant epithelia in vitro but the concentrations of $10-20 \mu \mathrm{m}$ used far exceed those achievable physiologically in humans. We utilised concentrations of statins with reduced PEC toxicity, allowing assessment of their potential role in invasion and early metastasis. The statin concentrations we used were higher than the serum levels detected with current statin dosing regimes, for example, peak plasma concentration of simvastatin in patients receiving $40 \mathrm{mg}$ per day is $7.2 \mathrm{~nm}\left(3 \mathrm{ng} \mathrm{ml}^{-1}\right)$ (Najib et al, 2003). However, in trials with lymphoma or myeloma patients (van der Spek et al, 2006), the maximum tolerated dose of simvastatin was $15 \mathrm{mg}$ per $\mathrm{kg}$ per day, which would increase the circulating serum levels reaching peripheral organs significantly. A regime of $45 \mathrm{mg}$ per $\mathrm{kg}$ per day lovastatin in patients with solid tumours induced peak serum levels $3.9 \mu \mathrm{m}$ (Thibault et al, 1996).

Using validated human BMS co-culture models (Hart et al, 2005), we have shown that lipophilic statins all affect the ability of PECs to invade towards and through BMS (Figures 1 and 3) to initiate and develop tumour colonies. BMS pretreatment (Figure 1A) had no effect on statins' ability to promote or inhibit this process. Figure 1B (PC-3 treatment alone) and Figure 1C (pretreatment of BMS and PC-3) showed similar reductions in invasion. Utilising the more complex model of metastasis using
BMECs and basement membrane, which recapitulates the blood BMS barrier, both rosuvastatin and simvastatin completely inhibited directed invasion towards BMS. These data suggest that statins affect PECs directly but have no effect on their microenvironment. The exact mechanism underlying statins' ability to block CaP migration is unknown but there are several possible aetiological factors.

A recent large cohort study of cancer-free males over the age of 35 , showed that men with desirable/borderline cholesterol levels $\left(<200 \mathrm{mg} \mathrm{dl}^{-1}-<240 \mathrm{mg} \mathrm{dl}^{-1}\right)$ had a lower risk of developing high grade $\mathrm{CaP}$ but no risk reduction in their overall incidence of $\mathrm{CaP}$ (Mondul et al, 2010). This supports other studies where low cholesterol was not associated with organ confined or low grade $\mathrm{CaP}$ but was associated with reduced risk of high grade cancer (Platz et al, 2008). A further epidemiological study also showed that low cholesterol was inversely associated with advanced disease $(\mathrm{OR}=0.42,95 \% \mathrm{CI}=0.13-1.36)$. The numbers in this study were small but the senior author (Platz et al, 2008) and others (Solomon and Freeman, 2008) have gone on to propose that statins affect CaP's intracellular cholesterol metabolism, which is known to be dysregulated. The uptake of statins by the liver and the requirement for liver-specific esterases, for example, in the case of the pro-drug simvastatin, limits statin availability in the peripheral circulation (Merck, 2005). It is therefore hypothesised that statins act by blocking HMG-CoA reductase in the liver and not in tumours, and that the reduction in cholesterol affects tumours at extrahepatic sites. Here we have shown that simvastatin can act directly on the PC3-GFP cells in the absence of activation, (Supplementary Figure 1) suggesting that simvastatin may act within the periphery. Cholesterol is an essential component of lipid rafts, cellular membrane microdomains that regulate signalling cascades originating from membrane-bound receptors such as tyrosine kinases. Treatment of $\mathrm{CaP}$ overnight or glioma cells for $48 \mathrm{~h}$ with simvastatin induced a $70 \%$ reduction in lipid raft cholesterol, an effect reversed by addition of exogenous cholesterol (Zhuang et al, 2005; Wu et al, 2009). This reduction inhibited the Aktl serinethreonine kinase/ protein kinase-B signalling pathway and induced apoptosis, an effect that could be reversed by cholesterol replenishment. Corroborating in vivo studies showed that elevated cholesterol levels increased pAkt1 signalling and promoted tumour growth, with a coincident reduction in apoptosis.

The studies above demonstrate a role of cholesterol in $\mathrm{CaP}$ progression by promoting tumour growth and preventing apoptosis. However, in the study presented here we are specifically modelling the effects of statins on the invasive and migratory abilities of $\mathrm{CaP}$ cell towards and within human BMS. Within these validated invasion coculture models, cholesterol was unable to recover the invasive phenotype after statin treatment. We have shown previously that invasion towards BMS occurs predominantly within the first $4 \mathrm{~h}$ (Hart et al, 2006). In this study, PC-3 cells were pretreated for $30 \mathrm{~min}$ before assay, suggesting that a reduction in cholesterol synthesis by $\mathrm{CaP}$ cells is not the factor inhibiting invasion. This observation is supported by co-culture assay data; the co-cultures were grown in the presence of FCS and horse serum, both of which contain cholesterol. It is also noteworthy that invasion was towards haematopoietically active adipocyte-rich human BMS, which contains high cholesterol levels. All the co-cultures treated with lipophilic statins showed proliferation of PC-3 cells but formed smaller colonies of tightly packed epithelial cells, differing in their morphology to the controls, losing the ruffled leading edges of their lamellipodia and displaying distorted trailing edges, indicating motility impairment. Taken together these models suggest that the inhibition of invasion towards/through BMS, but not proliferation, is not related directly to circulating and local tissue cholesterol levels.

A critical factor may be the impairment of the transmigrational mechanisms of cancer cells by the effects of statins, in particular, their effect on the mevalonate and isoprenoid axis. Tumour cell exposure to statins downregulates mevalonate expression through 
HMG-CoA reductase inhibition. This affects membrane integrity, cell signalling, protein synthesis and cell cycle progression. Disruption of these processes by statins may result in inhibition of tumour initiation, growth and metastasis (Boudreau et al, 2007). Mevalonate is the precursor of the isoprenoids FPP and GGPP, essential for the translocation of signalling proteins, such as the G-proteins Ras and Rho to the plasma membrane, where they mediate signal transduction by controlling cell differentiation, proliferation and migration (Hager et al, 2006). Addition of GGPP, unlike cholesterol or FPP, to our invasion assays completely restores the ability of PC-3 cells to invade towards the BMS, suggesting that the lipophilic statins act by preventing the translocation of nascent signalling proteins, which require geranylgeranylation, to the cell surface. This would lead to a reduction of signalling proteins at the cell surface and thereby preventing the cell from detecting the invasive stimulus from the BMS or BMS adipocytes (Brown et al, 2010).

Further studies support the notion that the mevalonate/ G-protein axis is important. Zhao et al (2010) demonstrated that $10 \mu \mathrm{M}$ lovastatin inhibited EGFR dimerisation and signalling via AKT and its downstream targets, 4E-binding protein 1 and S6 kinase 1, in a GGPP-dependent manner. Lovastatin also induced cytoskeletal disorganisation associated with increased inactive RhoA expression, which lacked the GGPP tag. Co-incubation with mevalonate or GGPP restored RhoA activity and EGF signalling. This suggests that inhibition of invasion towards and through BMS is more likely to arise from isoprenoid loss rather than loss of endogenous cholesterol production by the $\mathrm{CaP}$ cells. This latter factor may be critical; the ability of the cancer cell to move is fundamental to metastasis and its inhibition prohibits metastatic $\mathrm{CaP}$ survival in BMS (Lang et al, 1998). In vitro studies have demonstrated the critical importance of the Rho/Rac axis in mesenchymal to amoeboid transition and invasion/migration (Sanz-Moreno et al, 2008); these steps are fundamental to progression in prostate and other cancers. Thus, important elements of metastatic behaviour are potentially inhibited by lipophilic statins and these anti-migrational effects alone may be responsible for the CaP-related observations presented herein and reported clinically (Platz et al, 2006; Gutt et al, 2010).

Here we have used validated models of malignant prostate epithelial invasion to examine the effects of statins on the migratory pathway in $\mathrm{CaP}$ metastasis. We have shown for the first time in this laboratory setting that statins act directly on malignant PEC and block the formation of GGPP from HMG-CoA. Loss of GGPP leads to a significant reduction in the ability of malignant PEC to invade towards and through BMS and so reduce their ability to form colonies within the BMS. This statin effect is not universal across all classes of statins as the hydrophilic statin pravastatin had no effect on the PC-3 cell line in our models. In summary, statins appear to act on two key components on $\mathrm{CaP}$ metastasis; reduce tumour growth, possibly through the reduction of cholesterol but also, as described here, through inhibiting geranylgeranyl prenylated pathways required for transendothelial migration and BMS invasion.

Supplementary Information accompanies the paper on British Journal of Cancer website (http://www.nature.com/bjc)

\section{REFERENCES}

Boudreau DM, Yu O, Miglioretti DL, Buist DS, Heckbert SR, Daling JR (2007) Statin use and breast cancer risk in a large population-based setting. Cancer Epidemiol Biomarkers Prev 16: 416-421

Breau RH, Karnes RJ, Jacobson DJ, McGree ME, Jacobsen SJ, Nehra A, Lieber MM, St Sauver JL (2010) The association between statin use and the diagnosis of prostate cancer in a population based cohort. J Urol 184: 494-499

Brown MD, Hart C, Gazi E, Gardner P, Lockyer N, Clarke N (2010) Influence of omega-6 PUFA arachidonic acid and bone marrow adipocytes on metastatic spread from prostate cancer. $\mathrm{Br} J$ Cancer 102: 403-413

Brown MD, Hart CA, Gazi E, Bagley S, Clarke NW (2006) Promotion of prostatic metastatic migration towards human bone marrow stoma by Omega 6 and its inhibition by Omega 3 PUFAs. Br J Cancer 94: 842-853

Campbell MJ, Esserman LJ, Zhou Y, Shoemaker M, Lobo M, Borman E, Baehner F, Kumar AS, Adduci K, Marx C, Petricoin EF, Liotta LA, Winters M, Benz S, Benz CC (2006) Breast cancer growth prevention by statins. Cancer Res 66: 8707-8714

Coutinho LH, Gilleece MH, de Wynter E, Will A, Testa NG (1993) Clonal and long-term cultures using human bone marrow. In Haemopoiesis: A Practical Approach, Testa NG, Molineux G (eds), pp 75-106. Oxford University Press: Oxford

Ferlay J, Shin HR, Bray F, Forman D, Mathers C, Parkin DM (2010) GLOBOCAN 2008 v1.2, Cancer Incidence and Mortality Worldwide: IARC CancerBase No. 10 (Internet). International Agency for Research on Cancer: Lyon, France, 2010

Garwood S (2010) Statins and cardiac surgery. J Cardiothorac Vasc Anesth 24: $909-912$

George NJ (1988) Natural history of localised prostatic cancer managed by conservative therapy alone. Lancet 1: 494-497

Gutt R, Tonlaar N, Kunnavakkam R, Karrison T, Weichselbaum RR, Liauw SL (2010) Statin use and risk of prostate cancer recurrence in men treated with radiation therapy. J Clin Oncol 28: 2653-2659

Hager MH, Solomon KR, Freeman MR (2006) The role of cholesterol in prostate cancer. Curr Opin Clin Nutr Metab Care 9: 379-385

Hart C, Clarke N, Brown M (2006) Analysis of prostate tumour cell invasion using BD FluoroBlok and FLUOstar OPTIMA. BMG Labtech Application Notes 144
Hart CA, Brown M, Bagley S, Sharrard M, Clarke NW (2005) Invasive characteristics of human prostatic epithelial cells: understanding the metastatic process. Br J Cancer 92: 503-512

Kotamraju S, Williams CL, Kalyanaraman B (2007) Statin-induced breast cancer cell death: role of inducible nitric oxide and arginase-dependent pathways. Cancer Res 67: 7386-7394

Koyuturk M, Ersoz M, Altiok N (2007) Simvastatin induces apoptosis in human breast cancer cells: p53 and estrogen receptor independent pathway requiring signalling through JNK. Cancer Lett 250: 220-228

Lang SH, Clarke NW, George NJ, Allen TD, Testa NG (1998) Interaction of prostate epithelial cells from benign and malignant tumor tissue with bone-marrow stroma. Prostate 34: 203-213

Loeb S, Kan D, Helfand BT, Nadler RB, Catalona WJ (2010) Is statin use associated with prostate cancer aggressiveness? BJU Int 105: $1222-1225$

Merck (2005) Information for Prescribers. Merck Sharp \& Dohme Ltd: Cramlington, Northumberland, UK

Mondul AM, Clipp SL, Helzlsouer KJ, Platz EA (2010) Association between plasma total cholesterol concentration and incident prostate cancer in the CLUE II cohort. Cancer Causes Control 21: 61-68

Murtola TJ, Tammela TL, Maattanen L, Huhtala H, Platz EA, Ala-Opas M, Stenman UH, Auvinen A (2010) Prostate cancer and PSA among statin users in the Finnish prostate cancer screening trial. Int J Cancer 127: 1650-1659

Najib NM, Idkaidek N, Adel A, Admour I, Astigarraga RE, Nucci GD, Alam SM, Dham R, Qumaruzaman (2003) Pharmacokinetics and bioequivalence evaluation of two simvastatin $40 \mathrm{mg}$ tablets (Simvast and Zocor) in healthy human volunteers. Biopharm Drug Dispos 24: 183-189

Platz EA, Clinton SK, Giovannucci E (2008) Association between plasma cholesterol and prostate cancer in the PSA era. Int J Cancer 123: $1693-1698$

Platz EA, Leitzmann MF, Visvanathan K, Rimm EB, Stampfer MJ, Willett WC, Giovannucci E (2006) Statin drugs and risk of advanced prostate cancer. J Natl Cancer Inst 98: 1819-1825

Sanz-Moreno V, Gadea G, Ahn J, Paterson H, Marra P, Pinner S, Sahai E, Marshall CJ (2008) Rac activation and inactivation control plasticity of tumor cell movement. Cell 135: 510-523 
Scott LJ, Clarke NW, George NJ, Shanks JH, Testa NG, Lang SH (2001) Interactions of human prostatic epithelial cells with bone marrow endothelium: binding and invasion. Br J Cancer 84: 1417-1423

Solomon KR, Freeman MR (2008) Do the cholesterol-lowering properties of statins affect cancer risk? Trends Endocrinol Metab 19: 113-121

Stancu C, Sima A (2001) Statins: mechanism of action and effects. J Cell Mol Med 5: 378-387

Thibault A, Samid D, Tompkins AC, Figg WD, Cooper MR, Hohl RJ, Trepel J, Liang B, Patronas N, Venzon DJ, Reed E, Myers CE (1996) Phase I study of lovastatin, an inhibitor of the mevalonate pathway, in patients with cancer. Clin Cancer Res 2: 483-491

van der Spek E, Bloem AC, van de Donk NW, Bogers LH, van der Griend R, Kramer MH, de Weerdt O, Wittebol S, Lokhorst HM (2006) Dose-finding study of high-dose simvastatin combined with standard chemotherapy in patients with relapsed or refractory myeloma or lymphoma. Haematologica 91: 542-545

Wu H, Jiang H, Lu D, Xiong Y, Qu C, Zhou D, Mahmood A, Chopp M (2009) Effect of simvastatin on glioma cell proliferation, migration, and apoptosis. Neurosurgery 65: 1087-1096; discussion 1096-1097

Zhao TT, Le Francois BG, Goss G, Ding K, Bradbury PA, Dimitroulakos J (2010) Lovastatin inhibits EGFR dimerization and AKT activation in squamous cell carcinoma cells: potential regulation by targeting rho proteins. Oncogene 29: 4682-4692

Zhuang L, Kim J, Adam RM, Solomon KR, Freeman MR (2005) Cholesterol targeting alters lipid raft composition and cell survival in prostate cancer cells and xenografts. J Clin Invest 115: 959-968

This work is published under the standard license to publish agreement. After 12 months the work will become freely available and the license terms will switch to a Creative Commons Attribution-NonCommercial-Share Alike 3.0 Unported License. 